\title{
Pacific
}

Journal of

Mathematics

\section{ON THE INCIDENCE CYCLES OF A CURVE: SOME GEOMETRIC INTERPRETATIONS}

LUCIANA RAMELLA 


\title{
ON THE INCIDENCE CYCLES OF A CURVE: SOME GEOMETRIC INTERPRETATIONS
}

\author{
LUCIANA RAMELLA
}

In this paper, we note that the incidence cycles of a seminormal curve $X$ intervene in the calculation of the arithmetic genus $p_{a}(X)$, of the algebraic fundamental group $\pi_{1}^{\mathrm{alg}}(X)$ and of the Picard group $\operatorname{Pic}(X)$ of $X$. Really we do not consider only seminormal curves, but more generally varieties obtained from a smooth variety by glueing a finite set of points.

0. Introduction. By a curve we mean a dimension 1 quasi-projective scheme over an algebraically closed field $k$.

Let $X$ be a connected reduced seminormal curve (see $[\mathbf{T}],[\mathbf{P}]$ and [D] for the definition and the geometric meaning of seminormality).

Let $X_{1}, \ldots, X_{n}$ be the irreducible components of $X$; the normalization $\bar{X}$ of $X$ is isomorphic to the disjoint union $\bigsqcup_{i=1}^{n} \bar{X}_{i}$ of the normalizations $\bar{X}_{i}$ of the curves $X_{i}$. Let $\pi: \bar{X} \rightarrow X$ denote the normalization morphism.

Let $P_{1}, \ldots, P_{m}$ be the singular points of $X$ and let $x_{1}, \ldots, x_{M}$ be the branches of $X \quad(x \in \bar{X}$ is a branch of $X$ over a singular point $P$ of $X$ if $\left.x \in \pi^{-1}(P)\right)$.

We define $\nu(X)=M-m-n+1$. In [R] one can find a geometric characterization of the number $\nu(X)$ in terms of the incidence cycles of $X$. One associates to the curve $X$ the graph $\Gamma$ whose vertices are $P_{1}, \ldots, P_{m}, X_{1}, \ldots, X_{n}$ and whose edges represent the $M$ branches of $X$ in this way: if $x_{r}$ is a branch over $P_{i}$ and $x_{r} \in \bar{X}_{j}$, an edge joining $P_{i}$ and $X_{j}$ is constructed. Any cycle of the graph $\Gamma$ associated to $X$ is said to be an incidence cycle of $X$.

In $[\mathbf{R}]$ it is proved that the graph $\Gamma$ associated to $X$ is connected, the number of the independent cycles of $\Gamma$ is $\nu(X)$ and $\Gamma$ contains cycles if and only if $X$ satisfies one of the following conditions:

(a) an irreducible component of $X$ is not locally unibranch,

(b) two irreducible components of $X$ meet in more than one point,

(c) $X$ contains polygons.

In the present paper we'll consider more generally a class of varieties $X$ of dimension $r \geq 1$ and we'll see that the number $\nu(X)$ 
intervenes in the calculus of the arithmetic genus $p_{a}(X)$, of the algebraic fundamental group $\pi_{1}^{\mathrm{alg}}(X)$ and of the Picard group $\operatorname{Pic}(X)$ of $X$.

By a variety we mean a reduced quasi-projective scheme over an algebraically closed field $k$.

Now we recall the definition of glueing of varieties and of $k$ algebras.

Definition 0.1. Let $X$ and $X^{\prime}$ be two varieties, let $x_{1}, \ldots, x_{M}$ be closed points of $X^{\prime}$ and let $P$ be a closed point of $X$. We say that $X$ is obtained from $X^{\prime}$ by glueing $x_{1}, \ldots, x_{M}$ over $P$ if there exists a morphism $f: X^{\prime} \rightarrow X$, called a glueing morphism, making cocartesian the following square:

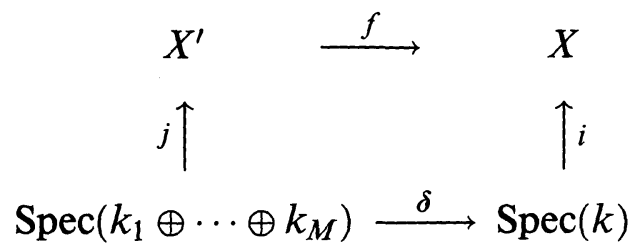

where $k_{i}$ is the residue field of $x_{i}$, the residue fields of $x_{i}$ and $P$ are isomorphic to $k, \delta$ is induced by the diagonal morphism, $i$ and $j$ are the canonical injections.

Algebraically Definition 0.1 is equivalent to the following

Definition 0.2 (see [T] $\S 1$ and [P] $\S 1$ ). Let $A$ and $B$ be two finitely generated $k$-algebras, with $k$ an algebraically closed field, let $\mathfrak{m}_{1}, \ldots, \mathfrak{m}_{M}$ be maximal ideals of $B$ and let $\mathfrak{m}$ be a maximal ideal of $A$. We say that $A$ is obtained from $B$ by glueing the maximal ideals $\mathfrak{m}_{1}, \ldots, \mathfrak{m}_{M}$ over $\mathfrak{m}$ if $A$ is the fibered product of $B$ and $k$ over $k_{1} \oplus \cdots \oplus k_{M}$, i.e. if we have the following cartesian square:

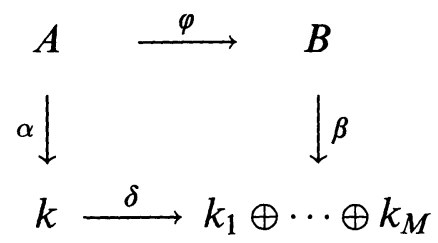

where $\alpha$ is the canonical projection $A \rightarrow A / \mathfrak{m} \cong k, \beta$ is the canonical projection $B \rightarrow B / \mathfrak{m}_{1} \oplus \cdots \oplus B / \mathfrak{m}_{M}=k_{1} \oplus \cdots \oplus k_{M}, k_{i} \cong k$ and $\delta$ is the diagonal morphism.

We recall that a seminormal curve $X$ is obtained from the normalization $\bar{X}$ by a finite number of glueing morphisms (see [T], Theorem 2.1). 
Note that Mestrano in [Me] used Severi curves, which are curves obtained from a finite (disjoint) union of projective lines by a finite number of glueing morphisms, to study the Picard group of the rational points of the Picard scheme of $C_{g}$, where $C_{g}$ is the universal curve over the function field of the coarse moduli space $M_{g}$ of the curves of genus $g$.

In what follows $X$ denotes a connected variety of pure dimension $r$ whose singular locus $\operatorname{Sing}(X)$ consists of a finite set of points $P_{1}, \ldots, P_{m}$, such that the normalisation $\bar{X}$ of $X$ is a smooth variety having $n$ connected components, every one of them of dimension $r, \bar{X}_{1}, \ldots, \bar{X}_{n}$ and the normalisation morphism $\pi: \bar{X} \rightarrow X$ is the composition of a finite number of glueing morphisms satisfying the conditions of Definition 0.1 . Let $M$ be the number of points of $\pi^{-1}(\operatorname{Sing}(X))$; we define $\nu(X)=M-m-n+1$.

We'll prove the following results:

THEOREM 0.3. If $X$ is projective, we have

$$
p_{a}(X)=p_{a}\left(\bar{X}_{1}\right)+\cdots+p_{a}\left(\bar{X}_{n}\right)+(-1)^{r-1} \nu(X) .
$$

THEOREM 0.4. We have

$$
\pi_{1}^{\mathrm{alg}}(X) \cong\left(\pi_{1}^{\mathrm{alg}}\left(\bar{X}_{1}\right) * \cdots * \pi_{1}^{\mathrm{alg}}\left(\bar{X}_{n}\right) * L_{\nu(X)}\right)^{-},
$$

where $L_{\nu}$ denotes the free group with $\nu$ generators, * denotes the free product of groups and $\wedge$ denotes the completion of the group.

THEOREM 0.5. We have $\operatorname{Pic}(X) \cong \operatorname{Pic}\left(\bar{X}_{1}\right) \oplus \cdots \oplus \operatorname{Pic}\left(\bar{X}_{n}\right) \oplus$ $\nu(X) k^{*}$, where $k^{*}$ is the multiplicative group $k-\{0\}$ and $\nu k^{*}$ denotes the direct sum of $\nu$ copies of $k^{*}$.

Theorem 0.3 is an easy calculation.

Theorem 0.4 was obtained by Vistoli in [V] for $X$ irreducible or having a unique singular point. He proved his result by obtaining any étale covering of $X$ from an étale covering of the normalization $\bar{X}$ by glueing the fibres of the branches of $X$.

By generalizing Vistoli's constructions described in [V], one can prove that any étale covering of $X$ is obtained from the étale coverings of $\bar{X}_{1}, \ldots, \bar{X}_{n}$ by a finite number of glueing morphisms.

But in a shorter way we'll prove Theorem 0.4 by induction on $n$ and by using Vistoli's results on varieties having only one singular point. 
Theorem 0.5 generalizes a result of Roberts contained in [Ro1] and in [Ro2]; by using the Mayer-Vietoris sequences, Roberts calculated the Picard group of an affine curve $X=\operatorname{Spec}(A)$ having the irreducible components $X_{i}$ rational, i.e. $\bar{X}_{i}=\operatorname{Spec}(k[t])$.

In order to calculate the Picard group $\operatorname{Pic}(X)$ of $X$, we construct the line bundles of $X$ by glueing line bundles of $\bar{X}$, by using a similar method as the one employed in [Mi] to construct the projective modules over a ring $A$ satisfying the conditions of Definition 0.2.

1. The arithmetic genus. The arithmetic genus of a projective variety $X$ of dimension $r$ is the number $p_{a}(X)=(-1)^{r}\left(\chi\left(O_{X}\right)-1\right)$, where $\chi\left(O_{X}\right)$ is the Euler-Poincare characteristic of $O_{X}$.

1.1. Proof of Theorem 0.3 . There is the following exact sequence of sheaves on $X: 0 \rightarrow O_{X} \rightarrow \pi_{*} O_{\bar{X}} \rightarrow \sum_{P \in X} \bar{O}_{X, P} / O_{X, P} \rightarrow 0$, where $\bar{O}_{X, P}$ is the integral closure of $O_{X, P}$. Since $O_{X, P}$ is obtained from $\bar{O}_{X, P}$ by glueing a finite number of maximal ideals, we have length $\left(\bar{O}_{X, P} / O_{X, P}\right)=M_{P}-1$, where $M_{P}$ is the number of points $x$ of $X$ lying over $P\left(x \in \pi^{-1}(P)\right)$. Since the morphism $\pi$ is affine, then $\chi\left(\pi_{*} O_{\bar{X}}\right)=\chi\left(O_{\bar{X}}\right)$ and therefore $\chi\left(O_{X}\right)=\chi\left(O_{\bar{X}}\right)-\sum_{P \in X}\left(M_{P}^{-1}\right)$.

Let us suppose $r$ odd.

We prove first that $p_{a}(\bar{X})=p_{a}\left(\bar{X}_{1}\right)+\cdots+p_{a}\left(\bar{X}_{n}\right)-n+1$. We proceed by induction on $n$. For $n=1$ it is true. Now we suppose that the statement is true for $n-1$ and we consider $Y=\bigsqcup_{i=1}^{n-1} \bar{X}_{i}$; then we have

$$
\begin{aligned}
p_{a}(\bar{X}) & =1-\chi\left(O_{\bar{X}}\right)=1-\chi\left(O_{\bar{X}_{n}}\right)-\chi\left(O_{Y}\right)=p_{a}\left(\bar{X}_{n}\right)+p_{a}(Y)-1 \\
& =p_{a}\left(\bar{X}_{1}\right)+\cdots+p_{a}\left(\bar{X}_{n}\right)-(n-1) .
\end{aligned}
$$

Then

$$
\begin{aligned}
p_{a}(X) & =1-\chi\left(O_{X}\right)=1-\chi\left(O_{\bar{X}}\right)+\sum_{P \in X}\left(M_{P}-1\right)=p_{a}(\bar{X})+M-m \\
& =p_{a}\left(\bar{X}_{1}\right)+\cdots+p_{a}\left(\bar{X}_{n}\right)+\nu(X) .
\end{aligned}
$$

If $r$ is even, the calculation is similar.

2. The algebraic fundamental group. If $X$ is connected, there exists a profinite topological group $G$ such that the category $\operatorname{Et}(X)$ of the étale coverings of $X$ is equivalent to the category $\operatorname{Ac}(G)$ of the finite sets on which $G$ acts continuously. $G$ is unique up to unique isomorphism; it is denoted $\pi_{1}^{\mathrm{alg}}(X)$ and it is defined the algebraic fundamental group of $X$.

Vistoli proved in [V] the following propositions: 
Proposition 2.1 (see [V], Teorema II.12). Let $X$ and $X^{\prime}$ be connected varieties and let $f: X^{\prime} \rightarrow X$ be a composition of a finite number of glueing morphisms; if $x \in X$, let $p(x)$ denote the cardinality of the fibre $f^{-1}(x)$.

We have $\pi_{1}^{\mathrm{alg}}(X)=\left(\pi_{1}^{\mathrm{alg}}\left(X^{\prime}\right) * L_{p}\right)^{\wedge}$, where $p=\sum_{x \in X}(p(x)-1)$.

Proposition 2.2 (see [V] Corollario II.11). Let $X_{1}, \ldots, X_{n}$ be disjoint connected varieties, let $x_{1} \in X_{1}, \ldots, x_{n} \in X_{n}$ be $n$ closed points. Let $X$ denote the variety obtained by glueing the points $x_{1}, \ldots, x_{n}$.

Then we have $\pi_{1}^{\mathrm{alg}}(X)=\left(\pi_{1}^{\mathrm{alg}}\left(X_{1}\right) * \cdots * \pi_{1}^{\mathrm{alg}}\left(X_{n}\right)\right)^{\text {^ }}$.

2.3. Proof of Theorem 0.4 . We proceed by induction on the number $n$ of the irreducible components of $X$. If $n=1$, the claim follows from Proposition 2.1.

Now we suppose that the theorem is true for $n-1$.

Let $X^{\prime}$ be the variety $\pi\left(\bigcup_{i=1}^{n-1} \bar{X}_{i}\right)$; we can suppose that $X^{\prime}$ is connected.

Furthermore we can suppose $P_{1} \in X^{\prime} \cap X_{n}$, so there exist a point $a \in X^{\prime}$ and a point $b \in X_{n}$ such that $\pi(a)=\pi(b)=P_{1}$. Let $X^{\prime \prime}$ denote the variety obtained from $X^{\prime} \sqcup X_{n}$ by glueing $a$ and $b$ over $P_{1}$.

The variety $X$ can be obtained from $X^{\prime \prime}$ by a finite number of glueing morphisms.

Then we can factor the morphism $\pi$ as:

$$
\pi: \bigsqcup_{i=1}^{n} \bar{X}_{i} \stackrel{\varphi_{1}}{\longrightarrow} X^{\prime} \sqcup \bar{X}_{n} \stackrel{\varphi_{2}}{\longrightarrow} X^{\prime} \sqcup X_{n} \stackrel{\varphi_{3}}{\longrightarrow} X^{\prime \prime} \stackrel{\varphi_{4}}{\longrightarrow} X .
$$

From the inductive hypothesis we have

$$
\pi_{1}^{\mathrm{alg}}\left(X^{\prime}\right)=\left(\pi_{1}^{\mathrm{alg}}\left(\bar{X}_{1}\right) * \cdots * \pi_{1}^{\mathrm{alg}}\left(\bar{X}_{n-1}\right) * L_{\nu\left(X^{\prime}\right)}\right)^{-} .
$$

From Proposition 2.2 we have $\pi_{1}^{\mathrm{alg}}\left(X^{\prime \prime}\right)=\left(\pi_{1}^{\mathrm{alg}}\left(X^{\prime}\right) * \pi_{1}^{\mathrm{alg}}\left(X_{n}\right)\right)^{\text {一 }}$ and from Proposition $2.1 \pi_{1}^{\mathrm{alg}}(X)=\left(\pi_{1}^{\mathrm{alg}}\left(X^{\prime \prime}\right) * L_{p}\right)^{\wedge}$, where $p=$ $\sum_{i=1}^{m} p\left(P_{i}\right)-m$ and $p\left(P_{i}\right)$ denotes the cardinality of the fibre $\varphi_{4}^{-1}\left(P_{i}\right)$.

We must prove $\nu(X)=\nu\left(X^{\prime}\right)+\nu\left(X_{n}\right)+p$.

If $\bar{Y}$ is a union of connected components of $\bar{X}$ and $Y=\pi(\bar{Y})$, we denote by $m_{Y}$ and $M_{Y}$ the number of the singular points of $Y$ and the number of the points of $\bar{Y}$ lying over the singular points of $Y$ respectively. We note that $\nu\left(X^{\prime}\right)=M_{X^{\prime}}-m_{X^{\prime}}-n+2$ and $\nu\left(X_{n}\right)=M_{X_{n}}-m_{X_{n}}$. 
Let us consider the last morphism $\varphi_{4}$; we find

$$
M=M_{X^{\prime \prime}}+\sum_{i=1}^{m} p\left(P_{i}\right)-m_{X^{\prime \prime}}
$$

Moreover the glueing morphism $\varphi_{3}$ gives the equalities $M_{X^{\prime \prime}}=$ $M_{X^{\prime}}+M_{X_{n}}+2$ and $m_{X^{\prime \prime}}=m_{X^{\prime}}+m_{X_{n}}+1$.

So, after easy calculations, we can conclude.

3. Line bundles obtained by glueing. We begin with a lemma.

LEMMA 3.1. Let $X$ be a (connected) quasi-projective variety and let $F$ be a locally free sheaf on $X$ of rank $r$. If $x_{1}, \ldots, x_{M}$ are $M$ closed points of $X$, then there exists an affine open $U$ of $X$ containing $x_{1}, \ldots, x_{M}$ such that the $O_{X}(U)$-module $F(U)$ is free of rank $r$.

Proof. For any (standard) affine open $V=\operatorname{Spec} A$ of $X$ we have that the sheaf $F_{\mid V}$ is isomorphic to the sheaf $\widetilde{N}$ associated to the $A$-module $N=F(V)$ (see [H], Chapter II, $\S 5$ ).

$N$ is a projective $A$-module of rank $r$ (see [Bo], Chapter II, $\S 5$, Theorem 1).

Let us choose $V$ containing the points $x_{1}, \ldots, x_{M}$; let $\mathfrak{m}_{1}, \ldots, \mathfrak{m}_{M}$ be the maximal ideals of $A$ corresponding to the points $x_{1}, \ldots, x_{M}$ respectively.

If $S=\bigcap_{i=1}^{M}\left(A-\mathfrak{m}_{i}\right)=A-\left(\bigcup_{i=1}^{M} \mathfrak{m}_{i}\right)$, the ring $A_{S}$ is semi-local, then the $A_{S}$-module $N_{S}=N \otimes_{A} A_{S}$ is free of rank $r$ (see [Bo], Chapter II, $\S 5$, Proposition 5) and there exists $f \in S$ such that $N_{f}$ is a free $A_{f}$-module (see [Bo], Chapter II, $\S 2$, Corollary 2 and the proof of the Proposition 2 of Chapter II, §5). We take $U=\operatorname{Spec} A_{f}$.

Let $X$ be a connected variety obtained from a variety $X^{\prime}$ by glueing the points $x_{1}, \ldots, x_{M}$ of $X^{\prime}$ over a point $P$ of $X$. The glueing morphism $f: X^{\prime} \rightarrow X$ induces a group homomorphism $f^{*}: \operatorname{Pic}(X) \rightarrow$ $\operatorname{Pic}\left(X^{\prime}\right)$. We want to see how a line bundle on $X$ originates from a line bundle on $X^{\prime}$.

In what follows we confuse a line bundle $L$ on $X$ with the locally free sheaf of rank 1 associated to it, but we denote by $L_{x}$ the fibre of the line bundle $L$ at the point $x \in X \quad\left(L_{x} \cong k\right)$ and by $L_{\mathfrak{m}}$ the fibre of the locally free sheaf $L$ at the point $x$ if $\mathfrak{m}$ denotes the maximal ideal of the local ring $O_{X, x}\left(L_{\mathfrak{m}} \cong O_{X, x}\right)$. 
Proposition 3.2 (We use the notations of Definition 0.1). Let $L$ be a line bundle on $X^{\prime}$. We have $j^{*}(L)=L_{x_{1}} \oplus \cdots \oplus L_{x_{M}}, L_{x_{i}} \cong k_{i} \cong k$, and let $h: L_{x_{1}} \oplus \cdots \oplus L_{x_{M}} \stackrel{\sim}{\rightarrow} k_{1} \oplus \cdots \oplus k_{M}$ be an isomorphism of $\left(k_{1} \oplus \cdots \oplus k_{M}\right)$-modules. Then the couple $(L, h)$ gives canonically a line bundle $L_{h}$ on $X$ such that $f^{*}\left(L_{h}\right)=L$.

Proof (see [Mi], §2). If $U$ is an affine open of $X$ containing $P$, we have $U=\operatorname{Spec}(A)$ and $f^{-1}(U)=\operatorname{Spec}(B), A$ and $B$ are two $k$-algebras satisfying the conditions of Definition 0.2.

Let $L_{h}(U)$ be the group fibred product of $k$ and $L\left(f^{-1}(U)\right)$ over $k_{1} \oplus \cdots \oplus k_{M}$, making cartesian the following square of groups:

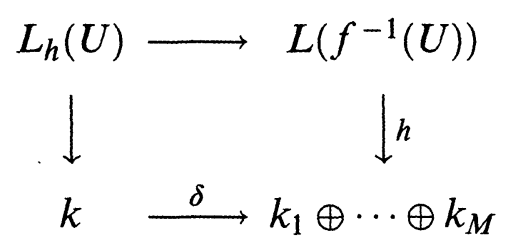

$L_{h}(U)$ is in a natural way an $A$-module and it is projective of rank 1.

If $U$ is an (affine) open of $X$ not containing $P$, we put $L_{h}(U)=$ $L\left(f^{-1}(U)\right)$. That defines a line bundle $L_{h}$ on $X$ (see [Bo], Chapter II, $\S 5$, Theorem 1) and we have $f^{*}\left(L_{h}\right)=L$.

Definition 3.3. (a) The couple $(L, h)$ of Proposition 3.2 is said to be the glueing of $L$ by $h$.

(b) Two glueings of line bundles $(L, h)$ and $\left(L^{\prime}, h^{\prime}\right)$ are said to be isomorphic if there exists an isomorphism $\lambda: L \rightarrow L^{\prime}$ such that the following diagram

$$
\begin{gathered}
L_{x_{1}} \oplus \cdots \oplus L_{x_{M}} \stackrel{h}{\longrightarrow} k_{1} \oplus \cdots \oplus k_{M} \\
\lambda \otimes 1_{k_{1} \oplus \cdots \oplus k_{M}} \downarrow \\
L_{x_{1}}^{\prime} \oplus \cdots \oplus L_{x_{M}}^{\prime} \stackrel{h^{\prime}}{\longrightarrow} k_{1} \oplus \cdots \oplus k_{M}
\end{gathered}
$$

is commutative.

(c) We define $(L, h) \cdot\left(L^{\prime}, h^{\prime}\right)=\left(L \otimes L^{\prime}, h \otimes h^{\prime}\right)$, where

$$
\left(h \otimes h^{\prime}\right)\left(u \otimes u^{\prime}\right)=h(u) h^{\prime}\left(u^{\prime}\right) .
$$

In this way the isomorphism classes of the couples $(L, h)$ form an abelian group $H_{f}$. 
Theorem 3.4. The Picard group $\operatorname{Pic}(X)$ of $X$ is isomorphic to the group $H_{f}$ defined as above.

Proof. We can define a natural group homomorphism $\Phi: H_{f} \rightarrow$ $\operatorname{Pic}(X)$ that to the class of $(L, h)$ associates the class of the line bundle $L_{h}$ constructed in the proof of Proposition 3.2, $\Phi$ is injective; in fact if $\Phi(L, h)=O_{X}$, we have that the couple $(L, h)$ is isomorphic to the couple $\left(O_{X}, \mathrm{id}_{k_{1} \oplus \cdots \oplus k_{M}}\right)$.

Now let $F$ be a line bundle on $X$. Then $L=f^{*}(F)$ is a line bundle on $X^{\prime}$ and from the square of Definition 0.1 , we see that $L_{x_{1}} \oplus \cdots \oplus L_{x_{M}}=j^{*}\left(f^{*}(F)\right)=\delta^{*}\left(i^{*}(F)\right)=\delta^{*}\left(F_{P}\right), F_{P} \cong k$.

$F$ induces an isomorphism $h: \delta^{*}\left(F_{P}\right) \stackrel{\sim}{\rightarrow} k_{1} \oplus \cdots \oplus k_{M}$. The couple $\left(f^{*}(F), h\right)$ gives with the above construction a line bundle over $X$ isomorphic to $F$ (see [Mi], §2). Hence $\Phi$ is surjective.

\section{The Picard group.}

Proposition 4.1. Let $f: X^{\prime} \rightarrow X$ be a glueing morphism of $M$ points $x_{1}, \ldots, x_{M}$ of a connected quasi-projective variety $X^{\prime}$ over a point $P$ of $X$. Then $\operatorname{Pic}(X) \cong \operatorname{Pic}\left(X^{\prime}\right) \oplus(M-1) k^{*}$.

Proof. It is sufficient to consider $M=2$. We'll prove the proposition by defining an isomorphism $\Psi$ from $H_{f}$ to $\operatorname{Pic}\left(X^{\prime}\right) \oplus k^{*}$ (cf. Theorem 3.4).

Let $L$ be a line bundle on $X^{\prime}$ and let $h$ be an isomorphism from $L_{x_{1}} \oplus L_{x_{2}}$ to $k_{1} \oplus k_{2}$. Let us consider an open affine $U$ of $X^{\prime}$ containing $x_{1}$ and $x_{2}$ such that there exists an isomorphism from $O_{X^{\prime}}(U)$ to $L(U)$ (see Lemma 3.1); let $e$ be the image of a unit $u$ of $O_{X^{\prime}}(U)$ satisfying the following condition:

$u$ is such that $\beta(u)$ is contained in the image of the diagonal morphism $\delta$ (see Definition 0.2).

$e_{i}=e \otimes 1_{k}$ is a generator of the $k$-vector space $L_{x_{i}}, i=1,2$. If $h\left(e_{1}, e_{2}\right)=(a, b)$, we define $\Psi((L, h))=\left(L, \frac{a}{b}\right)$.

We note that if $V$ and $e^{\prime}$ are an affine open of $X^{\prime}$ and a generator of $L(V)$ respectively satisfying the same conditions that $U$ and $e$ satisfy respectively, then we have $e^{\prime}=c e$, where $c$ is a unit of $O_{X^{\prime}}(U)$ satisfying the condition (*). Then $h\left(e_{1}^{\prime}, e_{2}^{\prime}\right)=h\left(\bar{c} e_{1}, \bar{c} e_{2}\right)=(\bar{c} a, \bar{c} b)$, $\bar{c} \in k^{*}$ and $\Psi((L, h))$ does not depend on the choice of $U$ and $e$.

If $(L, h)$ is isomorphic to $\left(L^{\prime}, h^{\prime}\right)$, there exists an isomorphism $\lambda$ 
from $L$ to $L^{\prime}$ such that $h\left(e_{1}, e_{2}\right)=h^{\prime}\left(e_{1}^{\prime}, e_{2}^{\prime}\right)$, where $e_{1}^{\prime}, e_{2}^{\prime}$ are the images in $L_{x_{1}}^{\prime}$ and $L_{x_{2}}^{\prime}$ respectively of $\lambda_{U}(e), \lambda_{U}$ is the isomorphism from $L(U)$ to $L^{\prime}(U)$ induced by $\lambda$. Then $\Psi((L, h))=\Psi\left(\left(L^{\prime}, h^{\prime}\right)\right)$.

It is easy to verify that the map $\Psi$ is a group isomorphism.

Proposition 4.2. Let $X^{\prime}$ be a quasi-projective variety having $n$ connected components $X_{1}, \ldots, X_{n}$, let $x_{i} \in X_{i}$ for every $i=1, \ldots, n$. Let $f: X^{\prime} \rightarrow X$ be the glueing morphism of the points $x_{1}, \ldots, x_{n}$. then $\operatorname{Pic}(X) \cong \operatorname{Pic}\left(X_{1}\right) \oplus \cdots \oplus \operatorname{Pic}\left(X_{n}\right)$.

Proof. We may assume $n=2$. From Theorem 3.4 it is sufficient to prove that the group $H_{f}$ is isomorphic to $\operatorname{Pic}\left(X^{\prime}\right) \cong \operatorname{Pic}\left(X_{1}\right) \oplus \operatorname{Pic}\left(X_{2}\right)$.

Let $L=L_{1} \oplus L_{2}$ be a line bundle on $X^{\prime}$. Let $U_{i}$ be an affine open of $X_{i}$ containing $x_{i}$, such that there exists an isomorphism $O_{X_{i}}\left(U_{i}\right) \rightarrow L_{i}\left(U_{i}\right)$, let $e_{i}$ denote the image of 1 , we denote the element $e_{i} \otimes 1_{k_{i}} \in L_{x_{i}}$ by $e_{i}$ also, $i=1,2$.

Let $i_{L}:\left(L_{1}\right)_{x_{1}} \oplus\left(L_{2}\right)_{x_{2}} \stackrel{\sim}{\rightarrow} k_{1} \oplus k_{2}$ denote the isomorphism defined by $i_{L}\left(e_{1}, e_{2}\right)=(1,1)$.

Two couples $\left(L^{\prime}, h\right)$ and $\left(L, i_{L}\right)$ of $H_{f}$ are isomorphic if and only if $L$ and $L^{\prime}$ are isomorphic; in fact, we can suppose $L^{\prime}=L$, if $h\left(e_{1}, e_{2}\right)=\left(a_{1}, a_{2}\right), a_{i}$ determines an isomorphism of $L_{i}$ into itself, $i=1,2$.

LEMMA 4.3. Let $f: X^{\prime} \rightarrow X$ be a morphism of connected quasiprojective varieties which is a composition of a finite number of glueing morphisms.

Let $\rho=\sum_{P \in X}(\rho(P)-1)$, where $\rho(P)$ is the cardinality of $f^{-1}(P)$.

Then $\operatorname{Pic}(X) \cong \operatorname{Pic}\left(X^{\prime}\right) \oplus \rho k^{*}$.

Proof. Let $P_{1}, \ldots, P_{m}$ be the points of $X$ having $\rho(P)>1$. We proceed by induction on $m$. If $m=1$ the result follows from Proposition 4.1 .

Now we suppose the lemma true for $m-1$. We can factor the morphism $f$ by $X^{\prime} \stackrel{f^{\prime}}{\rightarrow} X^{\prime \prime} \stackrel{f^{\prime \prime}}{\rightarrow} X$, where $f^{\prime}$ is the composition of the glueing morphisms over the points $P_{1}, \ldots, P_{m-1}$ only and $f^{\prime \prime}$ is the glueing over $P_{m}$.

By the induction hypothesis we have $\operatorname{Pic}\left(X^{\prime \prime}\right) \cong \operatorname{Pic}\left(X^{\prime}\right) \oplus \rho^{\prime} k^{*}$, $\rho^{\prime}=\sum_{P \in X^{\prime \prime}}\left(\rho^{\prime}(P)-1\right)$, where $\rho^{\prime}(P)$ is the cardinality of $f^{\prime-1}(P)$. By Proposition 4.1 we have $\operatorname{Pic}(X) \cong \operatorname{Pic}\left(X^{\prime \prime}\right) \oplus\left(\rho^{\prime \prime}\left(P_{m}\right)-1\right) k^{*}, \rho^{\prime \prime}\left(P_{m}\right)$ is the cardinality of $f^{\prime \prime-1}\left(P_{m}\right)$. 
4.4. Proof of Theorem 0.5. By using Proposition 4.2 and Lemma 4.3 , we can proceed by induction on the number $n$ of the irreducible components of $X$ as in the proof of Theorem 2 .

\section{REFERENCES}

[BM] H. Bass and P. Murty, Grothendieck groups and Picard groups of abelian group rings, Ann. of Math., 86 (1967), 16-73.

[Bo] N. Bourbaki, XXVII Algèbre Commutative Ch. II, Hermann, Paris, 1961.

[D] E. Davis, On the geometric interpretation of seminormality, Proc. Amer. Math. Soc., 68 (1978), 1-5.

[GRW] S. Geller, L. Reid and C. Weibel, The cyclic homology and K-theory of curves, J. Reine Angew. Math., 393 (1983), 39-90.

[Gr] A. Grothendieck, Revêtements étales et groupe fondamental, Lecture Notes in Math., vol. 224, Springer, Berlin-New York (1971).

[H] R. Hartshorne, Algebraic Geometry, Graduate texts in mathematics, vol. 52, Springer-Verlag, 1977.

[Me] N. Mestrano, Conjecture de Franchetta forte, Invent. Math., 87 (1987), 365376.

[Mi] J. Milnor, Introduction to Algebraic $K$-theory, Princeton University Press, 1971.

[P] C. Pedrini, Incollamenti di ideali primi e gruppi di Picard, Rend. Sem. Mat. Univ. Padova, 48 (1973), 39-66.

[R] L. Ramella, A geometric interpretation of one-dimensional quasinormal rings, J. Pure Appl. Algebra, 35 (1985), 77-83.

[Ro1] L. Roberts, The $K$-theory of some reducible affine varieties, J. Algebra, 35 (1975), 516-527.

[Ro1] _ The K-theory of some reducible affine curves: A combinatorial approach, in Algebraic K-theory, Lecture Notes in Math., vol. 551, SpringerVerlag, Berlin-New York (1976).

[T] C. Traverso, Seminormality and Picard group, Ann. Scuola Norm. Sup. Pisa, 24 (1970), 585-585.

[V] A. Vistoli, Incollamento di punti chiusi e gruppo fondamentale algebrico $e$ topologico, Rend. Sem. Mat. Univ. Padova, 69 (1983), 243-256.

Received May 4, 1991 and in revised form February 12, 1992.

Dipartimento di MATEMATICA-UNiversità

VIA L. B. Alberti 4

I-16132 Genova, Italy 


\title{
PACIFIC JOURNAL OF MATHEMATICS
}

\author{
Founded by
}

E. F. BECKENBACH (1906-1982) F. WoLF (1904-1989)

\section{EDITORS}

$\begin{array}{ll}\begin{array}{l}\text { V. S. VARADARAJAN } \\ \text { (Managing Editor) }\end{array} & \begin{array}{l}\text { NiCHOLAS ERCOLANI } \\ \text { University of Arizona }\end{array} \\ \text { University of California } & \text { Tucson, AZ 85721 } \\ \text { Los Angeles, CA 90024-1555 } & \text { ercolani@math.arizona.edu } \\ \text { vsv@math.ucla.edu } & \text { R. FinN } \\ \text { HERBERT CLEMENS } & \text { Stanford University } \\ \text { University of Utah } & \text { Stanford, CA 94305 } \\ \text { Salt Lake City, UT 84112 } & \text { finn@gauss.stanford.edu } \\ \text { clemens@math.utah.edu } & \text { VAUGHAN F. R. JoNEs } \\ \text { F. MichAEL CHRIsT } & \text { University of California } \\ \text { University of California } & \text { Berkeley, CA 94720 } \\ \text { Los Angeles, CA 90024-1555 } & \text { vfr@math.berkeley.edu } \\ \text { christ@math.ucla.edu } & \text { STEVEN KERCKHoFF } \\ \text { THomas ENRIGHT } & \text { Stanford University } \\ \text { University of California, San Diego } & \text { Stanford, CA 94305 } \\ \text { La Jolla, CA 92093 } & \text { spk@gauss.stanford.edu } \\ \text { tenright@ucsd.edu } & \end{array}$

\author{
C. C. MOORE \\ University of California \\ Berkeley, CA 94720
}

MaRTin ScharlemanN

University of California

Santa Barbara, CA 93106

mgscharl@henri.ucsb.edu

\author{
HAROLD STARK \\ University of California, San Diego \\ La Jolla, CA 92093
}

\begin{tabular}{ll}
\multicolumn{1}{c}{ SUPPORTING } & INSTITUTIONS \\
UNIVERSITY OF ARIZONA & UNIVERSITY OF OREGON \\
UNIVERSITY OF BRITISH COLUMBIA & UNIVERSITY OF SOUTHERN CALIFORNIA \\
CALIFORNIA INSTITUTE OF TECHNOLOGY & STANFORD UNIVERSITY \\
UNIVERSITY OF CALIFORNIA & UNIVERSITY OF HAWAII \\
MONTANA STATE UNIVERSITY & UNIVERSITY OF TOKYO \\
UNIVERSITY OF NEVADA, RENO & UNIVERSITY OF UTAH \\
NEW MEXICO STATE UNIVERSITY & WASHINGTON STATE UNIVERSITY \\
OREGON STATE UNIVERSITY & UNIVERSITY OF WASHINGTON
\end{tabular}

The Supporting Institutions listed above contribute to the cost of publication of this Journal, but they are not owners or publishers and have no responsibility for its content or policies.

Mathematical papers intended for publication in the Pacific Journal of Mathematics should be in typed form or offset-reproduced (not dittoed), double spaced with large margins. Please do not use built up fractions in the text of the manuscript. However, you may use them in the displayed equations. Underline Greek letters in red, German in green, and script in blue. The first paragraph must be capable of being used separately as a synopsis of the entire paper. In particular it should contain no bibliographic references. Please propose a heading for the odd numbered pages of less than 35 characters. Manuscripts, in triplicate, may be sent to any one of the editors. Please classify according to the 1991 Mathematics Subject Classification scheme which can be found in the December index volumes of Mathematical Reviews. Supply name and address of author to whom proofs should be sent. All other communications should be addressed to the managing editor, or Julie Speckart, University of California, Los Angeles, California 90024-1555.

There are page-charges associated with articles appearing in the Pacific Journal of Mathematics. These charges are expected to be paid by the author's University, Government Agency or Company. If the author or authors do not have access to such Institutional support these charges are waived. Single authors will receive 50 free reprints; joint authors will receive a total of 100 free reprints. Additional copies may be obtained at cost in multiples of 50 .

The Pacific Journal of Mathematics (ISSN 0030-8730) is published monthly except for July and August. Regular subscription rate: $\$ 190.00$ a year (10 issues). Special rate: $\$ 95.00$ a year to individual members of supporting institutions.

Subscriptions, orders for numbers issued in the last three calendar years, and changes of address should be sent to Pacific Journal of Mathematics, P.O. Box 969, Carmel Valley, CA 93924, U.S.A. Old back numbers obtainable from Kraus Periodicals Co., Route 100, Millwood, NY 10546.

The Pacific Journal of Mathematics at P.O. Box 969, Carmel Valley, CA 93924 (ISSN 0030-8730) is published monthly except for July and August. Second-class postage paid at Carmel Valley, California 93924, and additional mailing offices. Postmaster: send address changes to Pacific Journal of Mathematics, P.O. Box 969, Carmel Valley, CA 93924.

PUBLISHED BY PACIFIC JOURNAL OF MATHEMATICS, A NON-PROFIT CORPORATION Copyright $(\mathcal{1} 1993$ by Pacific Journal of Mathematics 


\section{PACIFIC JOURNAL OF MATHEMATICS}

Volume $157 \quad$ No. $2 \quad$ February 1993

Strong integral summability and the Stone-Čech compactification of the half-line

JEFF CONNOR and MARY ANNE SWARDSON

The endlich Baer splitting property

225

THEODORE GERARD FATICONI

The formal group of the Jacobian of an algebraic curve

MARGARET N. FREIJE

Concordances of metrics of positive scalar curvature

PAWEL GAJER

Explicit construction of certain split extensions of number fields and constructing cyclic classfields

STANLEY JOSEPH GURAK

Asymptotically free families of random unitaries in symmetric groups

ALEXANDRU MiHAi NiCA

On purifiable subgroups and the intersection problem

TAKASHI OKUYAMA

On the incidence cycles of a curve: some geometric interpretations

LUCIANA RAMELLA

On some explicit formulas in the theory of Weil representation

R. RANGA RAO

An analytic family of uniformly bounded representations of a free product of 373 discrete groups

JANUSZ WYSOCZAŃSKI

Errata: "Dentability, trees, and Dunford-Pettis operators on $L_{1}$ "

MARIA GIRARDI and ZHIBAO HU

Errata: "Poincaré cobordism exact sequences and characterisation" 